\title{
A Lymph Node Classification-based cancer-specific Survival Predicting Model in Patients with Ampullary cancer: A Derivation and Validation Study
}

\section{Jixiang Zhao}

First Affiliated Hospital of Nanchang University

\section{Yunzhi Zou}

First Affiliated Hospital of Nanchang University

\section{Xin Huang}

First Affiliated Hospital of Nanchang University

\section{Xiong Zhou}

First Affiliated Hospital of Nanchang University

\section{Xiaojiang Zhou}

First Affiliated Hospital of Nanchang University

Conghua Song ( $\nabla$ kesongs@email.nuc.edu.cn )

First Affiliated Hospital of Nanchang University https://orcid.org/0000-0002-1908-0640

\section{Research}

Keywords: Ampullary cancer, SEER, Derivation and validation study, Survival predicting model, TNM Stage

Posted Date: March 23rd, 2021

DOI: https://doi.org/10.21203/rs.3.rs-344928/v1

License: (c) (1) This work is licensed under a Creative Commons Attribution 4.0 International License. Read Full License 


\section{Abstract}

Background: Current lymph node $(\mathrm{LN})$ staging is controversial in predicting the survival of ampullary cancer (AC). We aimed to develop an alternative LN-classification-based nomogram to individualize AC prognosis.

Methods: Using the data of patients diagnosed with AC between 2004 and 2015 from the SEER database, we determined the cut-off values for the number of LNs examined via the K-adaptive partitioning algorithm. A nomogram predicting the survival of AC patients was performed, internally and externally validated, and evaluated by calibration plot, C-index, and decision curve analysis (DCA), and was compared to the $7^{\text {th }}$ TNM stage.

Results: We included 2341 patients with detailed information. The optimal cut-off for examined LN number was 12, while the cut-off value for positive LNs was 0 and 4 . The C-index for the nomogram was higher than that of the $7^{\text {th }}$ TNM staging (internal: $0.686 ; 95 \% \mathrm{Cl}, 0.584-0.773$ vs. $0.616 ; 95 \% \mathrm{Cl}, 0.533-$ $0.754, \mathrm{P}<0.001$; external: $0.713 ; 95 \% \mathrm{Cl}, 0.651-0.784$ vs. $0.647 ; 95 \% \mathrm{Cl}, 0.551-0.719, \mathrm{P}<0.001)$. Additionally, the nomogram showed good agreement between internal and external validation. DCA analysis showed no matter in the internal cohort or external cohort; the nomogram showed a greater benefit across the period of follow-up than did the $7^{\text {th }}$ TNM stage.

Conclusion: We found that examined LNs that were more than 12 were beneficial for prognosis of patients. We also modified the current $\mathrm{N}$ staging into three groups based on number of metastatic LNs: N0, no LN metastasis; N1, 1-4 metastatic LNs; N2, >=5 metastatic LNs. A nomogram with greater benefit for predicting the survival of patients with $A C$ than TNM staging was constructed.

\section{Introduction}

Ampullary cancer (AC) which accounts for $0.2 \%$ of all gastrointestinal tumors is a rare malignant tumor derived from the ampulla of vater ${ }^{1}$. According to an earlier report, the incidence was about one per 100 000 , whereas the newly diagnosed cases have obviously increased in the recent years based on data from the surveillance, epidemiology, and end results (SEER) ${ }^{1,2}$. Compared to pancreatic tumors, AC has a better survival rate, with a 5 -year survival rate of $30-60 \%{ }^{3}$. Several studies have demonstrated that some clinicopathological factors such as histology, tumor size and lymph node metastasis (LNM) are associated with the survival of patients with $A C^{1,3,4}$. With respect to histology, for instance, patients with the pancreatobiliary subtype have a poorer 5-year survival rate than those with the intestinal type $27.5 \%$ vs. 61\%), while patients with the mixed type almost have an intermediate survival rate1,3. Of these factors, lymph node (LN) involvement has been taken as a well-demonstrated factor correlated with the prognosis of patients with $\mathrm{AC}^{5}$. However, there are some controversial issues about the number of examined LNs and the category of positive LNs. Nassour et al stated that at least 17 LNs should be examined for optimal LN staging ${ }^{6}$, while J. Kwon thought it was of great benefit when the total number of LNs was up to $12^{7}$. Other studies also found that the number of LNs ranged from 12 to $16^{8,9,10}$. In 
addition, the category of positive LNs was also different among the suggestions proposed by several studies. Kang et al found that metastatic LNs were divided into three groups, namely $0,1-2$ and $\geq 3$ positive $L N s^{8}$, while Sakata $\mathrm{J}$ considered that the cut-off value of positive LNs was 0 and $3^{10}$. The 7 th edition of the AJCC cancer staging suggested that metastatic LNs staging was categorized as absent and present ${ }^{11}$. Also, some studies suggested that the $L N$ ratio was a good predictive factor and the value was $0.15-0.23^{5,8,12}$. Previous studies have demonstrated that patients with node-positive, pancreatobiliary type had a 5 -year survival rate of $20 \%$, while node-negative patients had a better prognosis with a 5 -year survival of $47 \%^{13}$. Based on these, we aimed to determine the optimal number of examined LNs and an alternative staging of the number of metastasis LNs by a large population-based study.

In our study, the best cut-off points were determined using the K-adaptive partitioning (KAPS) algorithm which was demonstrated as a useful tool to obtain heterogeneous subgroups by survival ${ }^{14}$. Through the SEER database, we developed and validated a nomogram to predict the survival of patients with AC.

\section{Methods}

\subsection{Patients}

The data of all patients with AC were retrieved from the SEER database with the National Cancer Institute's SEER`Stat software (version 8.3.6). Informed consent was not required because the SEER database is free for public use. According to the International Classification of Diseases in Oncology (ICD0-3), tumors with codes C24.1 were identified as AC. In our study, patients with AC were included according to the following criteria: (1) patients older than 20 years who were diagnosed as having AC by positive histology from 2004 through 2015; (2) patients with a detailed record on cancer-specific survival; (3) patients who were recorded with T stage, N stage, and M stage information; and (4) patients with detailed information, including race, grade, examined LNs, tumor size, and positive LNs.

\subsection{Clinicopathological factors}

The clinicopathological variables extracted from the SEER database in our study included age, race, sex, pathology grade, LNM, M stage, tumor size, $T$ stage, $\mathrm{N}$ stage, examined $\mathrm{LNs}$, and positive LNs. The patients were divided into three age groups: $<50$ years, $50-70$ years, and $\geq 70$ years. Race was classified into three types: white, black, and other. Sex included male and female. Pathology grade was categorized as the well/moderately differentiated type and poorly differentiated/undifferentiated type. LNM was described as N1 (Yes) or N0 (No). M1 (Yes) indicated a positive M stage. Tumor size was categorized into four groups: $\leq 2 \mathrm{~cm}, \leq 3 \mathrm{~cm}, \leq 5 \mathrm{~cm}$, and $>5 \mathrm{~cm}$. With respect to the examined nodes count, according to the results of the KAPS algorithm ${ }^{14}$, the cut-off was 12 . Therefore, regional nodes examined were divided into two groups: $\leq 12$ and $>12$. While the cut-off value of positive LNs was 0 and 4 , it was divided into three groups: $0,1-4$, and $\geq 5$. As for the KAPS algorithm, Soo-Heang Eo et al ${ }^{15}$. Proposed the use of a multi-way split in order to afford an optimal set of cut-off points: the optimal number of groups $(K)$ was 
found by a resampling technique which was implemented into an $\mathrm{R}$ package that we called KAPS. Simply, the code was available in the previous paper (kaps (formula, data, $K=2: 4$, mindat, type =c("perm", "NULL"), ...)). In our study, the main observation indicators were cancer-specific survival (CSS). CSS was defined as death attributable to this cancer.

\subsection{Statistical analysis}

For basic statistical analysis, patients were divided into two groups: the internal cohort included patients diagnosed with EC between 2004 and 2009, while the external validation cohort included those diagnosed between 2010 and 2015. Pearson's chi-squared test was used to investigate the associations between the categorical variables. To explore the potential risk factors for examined LNs, we performed univariate and multivariate logical regression, and we present the results as the odds ratio (OR) with the $95 \%$ confidence interval $(\mathrm{Cl})$. With respect to CSS of patients who were separated by examined LNs and positive LNs, we performed survival curves using the survminer package in $\mathrm{R}$ software. Furthermore, to analyze the related risk factors for survival, we performed multivariate Cox regression, and we determined the variables to perform nomograms according to Akaike Information Criterion (AIC). Finally, we selected CSS as the outcome of interest and performed nomogram based on the multivariate regression analysis. In addition, ROC curve, Calibration plot and DCA were used to assess the validity of the nomogram we constructed. All statistical analyses were performed with the R software (version 3.6.1, StataCorp LLC, College Station, Texas). The main packages used in our study included ggplot2, survival, rms, kaps, and survminer package. The chi-squared test was performed using SPSS (version 24.0). The results were considered to be statistically significant when the $P$ value $<0.05$.

\section{Results}

\subsection{Patients Characteristics}

As depicted by Supplementary Fig. 1, we enrolled 4990 patients for further investigation; of these, 2341 patients were died due to CSS, and 773 patients died due to other causes. In our evaluations, we considered CSS as the main outcome measure, and hence, we determined 2341 patients to explore prognostic factors. Detailed information of the selected patients is shown in the Table 1. As shown in the Table 1, we found that the age at diagnosis ranged from 50 to 70 years, and black people accounted for the lowest proportion of patients compared to white people (6.84\% vs. $77.93 \%)$. Interestingly, the pathology grade of AC was superior to moderately and poorly differentiated grades, while the rate of LNM reached $50 \%$. In addition, the tumor size tended to be smaller ( $\leq 5 \mathrm{~cm}$ vs. $>5 \mathrm{~cm}, 92.95 \%$ vs. $7.05 \%$ ), whereas AC was diagnosed at advanced stages ( $85.7 \%$ vs. $14.3 \%)$. In the subgroups divided by years, we found that the examined LNs and T stage were significantly different between the two groups.

\subsection{Grouping of LNs in Patients with AC}

We applied the KAPS algorithm to determine the categories of examined LNs and positive LNs. The cutoff value of examined LNs was 12, while the values of positive LNs were 0 and 4 (Table 1). As illustrated 
by Fig. 1, the Kaplan-Meier survival curve was performed according to the examined LNs and showed that there was an obvious difference between the two groups $(P=0.0014$, Fig. $1 \mathrm{~A})$. Patients with more than 12 examined LNs have better prognosis than those with less than 12 examined LNs. In addition, with respect to the category of positive LNs, we found that patients with 5 positive LNs had a poorer survival than the other two groups $(P<0.0001$, Fig. 1B), suggesting that the optimal groups of metastatic LNs were N0 (no positive $L N$ ), N1 (1-4 positive $L N s$ ) and N2 ( $\geq 5$ positive $L N s)$. To investigate the factors associated with the count of harvested LNs, we performed univariate and multivariate logistic regression analyses (Table 2). The results showed that patients diagnosed from 2010 to 2015 tended to have more examined LNs compared to those who were diagnosed from 2004 to $2009(P=0.000)$. Patients with bigger tumor sizes were associated with more harvested LNs $(P=0.018)$. As expected, patients with advanced tumors were inclined to have more LNs harvested. In a nutshell, year of diagnosis, tumor size and T stage were identified as independent factors associated with harvested LNs.

\subsection{AC Survival Prediction Model}

To construct a survival prediction model, we first performed a multivariate Cox regression analysis and then built a nomogram plot. As listed in Table 3, patients with age $>70$ years, advanced T stage, distant metastasis, positive LNM and poorly differentiated had a poorer prognosis, while patients with more examined LNs were correlated with better prognosis. As for building the nomogram, the positive LNs factor was not included due to the fact that the value of AIC was obviously larger when it was enrolled in the nomogram. Therefore, we established a nomogram based on other five prognostic factors (Fig. 2). According to the nomogram, we found that T stage contributed the most to the prognosis of patients with $\mathrm{AC}$, followed by M stage, then pathological grade and age, whereas the examined LNs had the least probability of predicting survival. As for the explanation of the nomogram, a straight line can be drawn down at each time point to determine the estimated probability of survival. With respect to each predictor, we could read the points assigned on a 0-10 scale at the top and then add these points. Finally, we read the corresponding predictions of 1-, 3-, and 5-year risks by finding the number on the "Total Points" scale.

\subsection{Validation in the internal and external cohort}

To investigate the validity of the nomogram, we divided the patients into internal and external cohorts according to year of diagnosis and determined the C-index value. As listed in Table 4, the value of C-index in the internal cohort was $0.686(95 \% \mathrm{Cl}, 0.584-0.773)$ and the bootstrap corrected value was 0.674 , both of which were higher than the value of TNM stage (C-index, $0.616,95 \% \mathrm{Cl}, 0.533-0.754$; bootstrap corrected, 0.615 ), suggesting that the nomogram was more effective for predicting survival than the TNM stage. In line with the results of the external cohort, the nomogram was superior to TNM stage (external cohort, $0.713,95 \% \mathrm{Cl}, 0.651-0.784$, bootstrap corrected, 0.698 ; TNM stage, $0.647,95 \% \mathrm{Cl}, 0.551-0.719$, bootstrap corrected, 0.643 ). We equally found that there was high consistency between actual and predicted values in the nomogram predicting model (Fig. 3). With respect to the specificity and sensitivity of the nomogram, in the internal cohort, we found that the values of area under the curve (AUC) predicting 1-year, 3-year and 5-year survivals by the nomogram were $0.719(0.634-0.781), 0.788(0.672-0.833)$ and $0.782(0.653-0.858)$, respectively, while the values of the TNM stage predicting model were 0.596 
(0.537-0.702), $0.673(0.601-0.714)$ and $0.689(0.633-0.768)$, respectively (Table 4). Compared to the TNM stage model, the nomogram was better at predicting the 1-year, 3-year and 5-year prognoses (Fig. 4A-4C). As indicted by the external cohort, the nomogram also performed better than the TNM stage model (1-year AUC: 0.698 vs. 0.658, 3-year AUC: 0.764 vs. 0.717, 5-year AUC: 0.774 vs. $0.677, P<0.001$ ) (Table 4 and Fig. 4D-4F). Furthermore, to compare the clinical usability between the nomogram and TNM stage models, we performed a DCA plot. As illustrated in Fig. 5, regardless of whether in the internal cohort or in the external cohort, the predicting efficiency of the nomogram was better than that of TNM stage for 1-year, 3-year and 5-year survivals.

\section{Discussion}

To our knowledge, ACs are tumors located at the junction of the distal bile duct, the main pancreatic duct and the duodenum. They are usually classified into pancreaticobiliary, intestinal and mixed tumors, by which some clinicopathological scores and nomograms have been established to evaluate prognosis ${ }^{3,16,17}$. However, these predicting models were not sufficient to evaluate the outcome of patients with AC. Therefore, some researchers thought that it would be more beneficial to include some biomarkers such as CK7, MUC1 and MUC5AC; however, this was controversial with regard to the prognostic value of the molecular alterations ${ }^{18,19,20}$. Moreover, too many prognostic factors would be needed to construct a significant model. Therefore, up to now, there is still no effective and suitable model for predicting prognosis. In our study, 2341 patients were extracted from the SEER database to investigate the prognostic factors of CSS. As for the basic characteristics of AC, consistent with other studies, we found that the age at diagnosis of patients with AC mainly ranged from 50-70 years, white people accounted for a majority of the patients and most of the tumors were smaller than $5 \mathrm{~cm}^{21,22}$. As for the LNM rate, we found that it was almost as high as the no LNM rate. Actually, it has been reported that the LNM rate of AC is high. John $R$ et al. reported that positive LNM could be found in $45 \%$ of T1 patients and $86 \%$ of $\mathrm{T} 4$ patients ${ }^{22}$.

As for the curative treatment of AC, it is estimated that more than $80 \%$ of ACs are amenable to resection including surgical and endoscopic removal. For LNM negative lesions, pancreaticoduodenectomy was an indeed curative treatment, while it had a better ability to provide indications for surgery for LNM positive lesions although some lesions were in the early stage ${ }^{23}$. In spite of some notions about the invasion of lymphatic vessels, LNM was an important factor for the determination of treatment methods and to predict prognosis ${ }^{24}$. Hence, the number of harvested LNs was the key factor to determine the optimal LNM state. In our study, we identified the best cut-off value by the KAPS algorithm which has been used in many studies ${ }^{14,25}$. In consistence with some studies ${ }^{8,11}$, we found that patients with $>12$ examined LNs have more benefit than those who with $<12$; however, some studies thought the harvested LNs should be $>17^{6,7}$. In addition, the multivariate logistic regression analysis showed that tumor size and $T$ stage were independent factors of harvesting LNs, in corroboration with the results of a previous study ${ }^{26}$. With respect to $\mathrm{N}$ stage, some studies proposed that the best cutoff point for positive LNs was 0 and $3^{10,27}$, while few studies thought that positive LNs should be divided into $0-1$ and $\geq 2$ groups $^{7}$. In our 
study, the new LNM stage we proposed was N0 (0 positive LNs), N1 (1-4 positive LNs) and N2 ( $\geq 5$ positive LNs), which made a great difference for survival. However, the positive LNs factor was not included in the construction of the nomogram plot because the value of AIC was too large. AIC was considered as an important criterion for variable sieving and has been used in many studies ${ }^{28,29}$. Compared with our model, the TNM staging system earlier was not better for survival prediction, while our model was reasonable and logic by multivariate cox regression analysis and AIC algorithm which can prevent over fitting of the model and ensure the accuracy of model ${ }^{30}$. In our model, we recruited age, T stage, $\mathrm{M}$ stage, pathology grade and examined lymph nodes other than TNM stage, avoiding the insufficiency of TNM stage. Naturally, nomogram we conducted performed better than TNM stage, which was demonstrated by ROC curve and DCA analysis.

Our study had some limitations that should be discussed. First, the TNM staging used was the 7th edition rather than the 8th edition, which may influence the results of the comparison between the nomogram and TNM staging models. Hence, it is necessary to perform studies to compare the model constructed by our study with the 8th edition of TNM staging. Second, we excluded many patients who had missing data associated with our collected variables, increasing the selection bias. Third, the variables including examined LNs and positive LNs were diagnosed depending on each doctor in different clinical centers. Finally, the enrolled prognostic parameters were so limited that we could not comprehensively analyze them. Therefore, although this nomogram performed well in the two cohorts, it should be applied with great caution when assessing the risk of 1-, 3- and 5-year survivals. In the future, we will collect our relevant data to incorporate the factors above into further research.

In conclusion, the present study was constructed to investigate the optimal examined and positive LNs. We found that the examined LNs factor was beneficial for the prognosis of the patients, which was more favorable with at least $12 \mathrm{LNs}$. We also modified the current $\mathrm{N}$ staging into three groups based on number of metastatic LNs: N0, no LN metastasis; N1, 1-4 metastatic LNs; and N2, $\geq 5$ metastatic LNs. Based on the categories and multivariate analysis, we developed and validated a nomogram with greater benefit for predicting the survival of patients with AC than the TNM staging, which was demonstrated by td-ROC and DCA.

\section{Declarations}

\section{Ethics approval and consent to participate:}

Ethics approval and consent was obtained from SEER database.

\section{Patient consent for publication:}

Not Appliable 


\section{Consent for publication:}

All study participants provided informed consent.

\section{Data Availability Statement:}

The datasets [ANALYZED] for this study can be found in the [Surveillance, Epidemiology, and End Results Program] [https://seer.cancer.gov/].

\section{Conflict of Interest}

The authors declare no conflict of interest related to this study.

\section{Funding:}

This work was supported by the National Natural Science Foundation of China (grant. no. 81860099).

\section{Author Contributions:}

C.S and Xj.Z contributed to the conception and design of the work. J.Z and Y.Z contributed to design, data analysis, editing the manuscript and critical revision of the manuscript. X.H contributed to data analysis. J.Z and X.Z contributed to data acquisition. All authors read and approved the final manuscript.

\section{Acknowledgments:}

We would like to thank Editage (www.editage.com) for English language editing.

\section{References}

1. Carter JT, Grenert JP, Rubenstein L, Stewart L, Way LW. Tumors of the ampulla of vater: histopathologic classification and predictors of survival. J Am Coll Surg. 2008;207(2):210-218. doi:10.1016/j.jamcollsurg.2008.01.028

2. Hester $C A$, Dogeas $E$, Augustine MM, et al. Incidence and comparative outcomes of periampullary cancer: A population-based analysis demonstrating improved outcomes and increased use of adjuvant therapy from 2004 to 2012. J Surg Oncol. 2019;119(3):303-317. doi:10.1002/jso.25336

3. Zimmermann C, Wolk S, Aust DE, et al. The pathohistological subtype strongly predicts survival in patients with ampullary carcinoma. Sci Rep. 2019;9(1):12676. doi:10.1038/s41598-019-49179-w 
4. Maithel SK, Khalili K, Dixon E, et al. Impact of regional lymph node evaluation in staging patients with periampullary tumors. Ann Surg Oncol. 2007;14(1):202-210. doi:10.1245/s10434-006-9041-9

5. Chen SC, Shyr YM, Chou SC, Wang SE. The role of lymph nodes in predicting the prognosis of ampullary carcinoma after curative resection. World J Surg Oncol. 2015;13:224. doi:10.1186/s12957015-0643-1

6. Nassour I, Christie A, Choti MA, et al. Determining the Adequate Examined Lymph Node Count in Resected Ampullary Adenocarcinoma-A National Cohort Study. J Gastrointest Surg. 2018;22(5):792801. doi:10.1007/s11605-018-3737-6

7. Kwon J, Kim K, Chie EK, et al. Prognostic relevance of lymph node status for patients with ampullary adenocarcinoma after radical resection followed by adjuvant treatment. Eur J Surg Oncol. 2017;43(9):1690-1696. doi:10.1016/j.ejso.2017.05.024

8. HJ K, SH E, SC K, et al. Increased number of metastatic lymph nodes in adenocarcinoma of the ampulla of Vater as a prognostic factor: a proposal of new nodal classification. Surgery. 2014;155(1):74-84. doi:10.1016/j.surg.2013.08.004

9. Falconi M, Crippa S, Domínguez I, et al. Prognostic relevance of lymph node ratio and number of resected nodes after curative resection of ampulla of Vater carcinoma. Ann Surg Oncol. 2008;15(11):3178-3186. doi:10.1245/s10434-008-0099-4

10. Sakata J, Shirai Y, Wakai T, Ajioka Y, Akazawa K, Hatakeyama K. Assessment of the nodal status in ampullary carcinoma: the number of positive lymph nodes versus the lymph node ratio. World $J$ Surg. 2011;35(9):2118-2124. doi:10.1007/s00268-011-1175-7

11. Washington K. 7th edition of the AJCC cancer staging manual: stomach. Ann Surg Oncol. 2010;17(12):3077-3079. doi:10.1245/s10434-010-1362-z

12. Partelli $S$, Crippa $S$, Capelli $P$, et al. Adequacy of lymph node retrieval for ampullary cancer and its association with improved staging and survival. World J Surg. 2013;37(6):1397-1404. doi:10.1007/s00268-013-1995-8

13. Zhou Y, Li D, Wu L, Si X. The histopathologic type predicts survival of patients with ampullary carcinoma after resection: A meta-analysis. Pancreatology. 2017;17(2):273-278. doi:10.1016/j.pan.2017.01.007

14. Wu S, Chen JN, Zhang QW, et al. A New Metastatic Lymph Node Classification-based Survival Predicting Model in Patients With Small Bowel Adenocarcinoma: A Derivation and Validation Study. EBioMedicine. 2018;32:134-141. doi:10.1016/j.ebiom.2018.05.022

15. Eo, Soo-Heang, Seung-Mo Hong and HYC. K-adaptive partitioning for survival data: The Kaps add-on package for R. arXiv Prepr arXiv. 1306.4615.

16. Al-Jumayli M, Batool A, Middiniti A, et al. Clinical Outcome of Ampullary Carcinoma: Single Cancer Center Experience. J Oncol. 2019;2019:3293509. doi:10.1155/2019/3293509

17. Huang XT, Huang CS, Chen W, et al. Development and validation of a nomogram for predicting overall survival of node-negative ampullary carcinoma. J Surg Oncol. 2020;121(3):518-523. doi:10.1002/jso. 25816 
18. Kulkarni MM, Khandeparkar SGS, Joshi AR, Kakade A, Fegade L, Narkhede K. Clinicopathological Study of Carcinoma of the Ampulla of Vater with Special Reference to MUC1, MUC2 and MUC5AC Expression. J Clin Diagn Res. 2017;11(5):Ec17-ec20. doi:10.7860/jcdr/2017/26842.9830

19. Perysinakis I, Minaidou E, Mantas D, et al. Differentiation and prognostic markers in ampullary cancer: Role of p53, MDM2, CDX2, mucins and cytokeratins. Pathol Res Pr. 2016;212(11):1039-1047. doi:10.1016/j.prp.2016.09.004

20. Sessa F, Furlan D, Zampatti C, Carnevali I, Franzi F, Capella C. Prognostic factors for ampullary adenocarcinomas: tumor stage, tumor histology, tumor location, immunohistochemistry and microsatellite instability. Virchows Arch. 2007;451(3):649-657. doi:10.1007/s00428-007-0444-1

21. Klein F, Jacob D, Bahra M, et al. Prognostic factors for long-term survival in patients with ampullary carcinoma: the results of a 15-year observation period after pancreaticoduodenectomy. HPB Surg. 2014;2014:970234. doi:10.1155/2014/970234

22. Hornick JR, Johnston FM, Simon PO, et al. A single-institution review of 157 patients presenting with benign and malignant tumors of the ampulla of Vater: management and outcomes. Surgery. 2011;150(2):169-176. doi:10.1016/j.surg.2011.05.012

23. Brown KM, Tompkins AJ, Yong S, Aranha G V, Shoup M. Pancreaticoduodenectomy is curative in the majority of patients with node-negative ampullary cancer. Arch Surg. 2005;140(6):523-529. doi:10.1001/archsurg.140.6.529

24. Hsu HP, Shan YS, Hsieh YH, Yang TM, Lin PW. Predictors of recurrence after pancreaticoduodenectomy in ampullary cancer: comparison between non-, early and late recurrence. J Formos Med Assoc. 2007;106(6):432-443. doi:10.1016/s0929-6646(09)60292-8

25. Guo W, Bao S, Lin W, et al. Estimating the size of HIV key affected populations in Chongqing, China, using the network scale-up method. PLoS One. 2013;8(8):e71796. doi:10.1371/journal.pone.0071796

26. Okano K, Asano E, Kushida Y, Kamada H, Mori H, Suzuki Y. Factors influencing lymph node metastasis in patients with ampullary adenocarcinoma. Dig Surg. 2014;31(6):459-467. doi:10.1159/000370251

27. Sakata J, Shirai Y, Wakai T, et al. Number of positive lymph nodes independently affects long-term survival after resection in patients with ampullary carcinoma. Eur J Surg Oncol. 2007;33(3):346-351. doi:10.1016/j.ejso.2006.10.008

28. You Y, YC S, DW C, et al. Proposed Modification of Staging for Distal Cholangiocarcinoma Based on the Lymph Node Ratio Using Korean Multicenter Database. Cancers (Basel). 2020;12(3). doi:10.3390/cancers12030762

29. Huh K, Hong J, Jung J. Association of meteorological factors and atmospheric particulate matter with the incidence of pneumonia: an ecological study. Clin Microbiol Infect. Published online 2020. doi:10.1016/j.cmi.2020.03.006

30. Fang J, Zhang B, Wang S, et al. Association of MRI-derived radiomic biomarker with disease-free survival in patients with early-stage cervical cancer. Theranostics. 2020;10(5):2284-2292. doi:10.7150/thno.37429 


\section{Tables}

Table 1

Patients' demographics, clinical characteristics at diagnosis 


\begin{tabular}{|c|c|c|c|c|}
\hline Variables & Total (\%) & 2004-2009 & 2010-2015 & P Value \\
\hline$n$ & 2341 & $965(48.25 \%)$ & $1376(51.75 \%)$ & \\
\hline Age & & & & 0.476 \\
\hline$<50$ & $264(11.28 \%)$ & $118(12.23 \%)$ & $146(10.61 \%)$ & \\
\hline $50-70$ & $1279(54.63 \%)$ & $522(54.09 \%)$ & 757(55.01\%) & \\
\hline$>70$ & 798(34.09\%) & $325(33.68 \%)$ & $473(34.38 \%)$ & \\
\hline Race & & & & 0.254 \\
\hline White & $1784(76.21 \%)$ & 752(77.93\%) & $1032(75 \%)$ & \\
\hline Black & $176(7.52 \%)$ & $66(6.84 \%)$ & $110(7.99 \%)$ & \\
\hline Other & $381(16.28 \%)$ & $147(15.23 \%)$ & $234(17.01 \%)$ & \\
\hline Sex & & & & 0.333 \\
\hline Female & $1013(43.27 \%)$ & $429(44.46 \%)$ & $584(42.44 \%)$ & \\
\hline Male & $1328(56.73 \%)$ & $536(55.54 \%)$ & $792(57.56 \%)$ & \\
\hline Pathology Grade & & & & 0.406 \\
\hline Well & $254(10.85 \%)$ & 101(10.47\%) & $153(11.12 \%)$ & \\
\hline Moderately differentiated & $1259(53.78 \%)$ & $506(52.44 \%)$ & $753(54.72 \%)$ & \\
\hline Poorly & $810(34.6 \%)$ & $352(36.48 \%)$ & $458(33.28 \%)$ & \\
\hline Undifferentiated & $18(0.77 \%)$ & $6(0.62 \%)$ & $12(0.87 \%)$ & \\
\hline Lymph node Metastasis & & & & 0.404 \\
\hline NO & $1041(44.47 \%)$ & $439(45.49 \%)$ & $602(43.75 \%)$ & \\
\hline Yes & $1300(55.53 \%)$ & $526(54.51 \%)$ & $774(56.25 \%)$ & \\
\hline Metastasis & & & & 0.296 \\
\hline No & 2278(97.31\%) & $935(96.89 \%)$ & $1343(97.6 \%)$ & \\
\hline Yes & $63(2.69 \%)$ & $30(3.11 \%)$ & $33(2.4 \%)$ & \\
\hline Tumor size & & & & 0.79 \\
\hline$\leq 2 \mathrm{~cm}$ & $1127(48.14 \%)$ & $470(48.7 \%)$ & $657(47.75 \%)$ & \\
\hline$<=3 \mathrm{~cm}$ & $620(26.48 \%)$ & $256(26.53 \%)$ & $364(26.45 \%)$ & \\
\hline$<=5 \mathrm{~cm}$ & $436(18.62 \%)$ & 171(17.72\%) & 265(19.26) & \\
\hline$>5 \mathrm{~cm}$ & $158(6.75 \%)$ & $68(7.05 \%)$ & $90(6.54 \%)$ & \\
\hline
\end{tabular}




\begin{tabular}{|lllll|}
\hline Examined LNs count & & & 0.000 \\
\hline$<=12$ & $1034(44.17 \%)$ & $527(54.61 \%)$ & $507(36.85 \%)$ & \\
\hline T stage & $1306(55.79 \%)$ & $438(45.39 \%)$ & $869(63.15 \%)$ & \\
\hline T1 & & & & 0.013 \\
\hline T2 & $292(12.47 \%)$ & $138(14.3 \%)$ & $154(11.19 \%)$ & \\
\hline T3 & $668(28.53 \%)$ & $247(25.6 \%)$ & $421(30.6 \%)$ & \\
\hline T4 & $737(31.48 \%)$ & $319(33.05 \%)$ & $418(30.38 \%)$ & \\
\hline Positive LNs count & $644(27.51 \%)$ & $261(27.05 \%)$ & $383(27.83 \%)$ & \\
\hline 0 & & & & 0.437 \\
\hline 1-4 & $1049(44.81 \%)$ & $445(46.11 \%)$ & $604(43.9 \%)$ & \\
\hline$>=5$ & $852(36.39 \%)$ & $349(36.17 \%)$ & $503(36.56 \%)$ & \\
\hline 7th TNM stage & $440(18.8 \%)$ & $171(17.72 \%)$ & $269(19.55 \%)$ & \\
\hline I & & & & 0.563 \\
\hline II & $604(25.8 \%)$ & $244(25.28 \%)$ & $360(26.16 \%)$ & \\
\hline III & $1055(45.06 \%)$ & $442(45.8 \%)$ & $613(44.55 \%)$ & \\
\hline IV & $618(26.39 \%)$ & $248(25.7 \%)$ & $370(26.89 \%)$ & \\
\hline & $64(2.73 \%)$ & $31(3.21 \%)$ & $33(2.4 \%)$ & \\
\hline
\end{tabular}

Table 2

Univariate and Multivariate logistic regression analysis of ampulla cancer patients for examined LNs 


\begin{tabular}{|c|c|c|c|c|}
\hline Variables & Univariate analysis & \multicolumn{3}{|c|}{ Multivariate analysis } \\
\hline \multicolumn{5}{|l|}{ Age } \\
\hline$<50$ & Reference & - & & \\
\hline $50-70$ & $0.909(0.694-1.19)$ & 0.487 & & \\
\hline$>70$ & $0.789(0.595-1.046)$ & 0.099 & & \\
\hline \multicolumn{5}{|l|}{ Race } \\
\hline White & Reference & - & & \\
\hline Black & $1.073(0.784-1.467)$ & 0.66 & & \\
\hline Other & $1.01(0.808-1.262)$ & 0.931 & & \\
\hline \multicolumn{5}{|l|}{ Sex } \\
\hline Male & Reference & - & & \\
\hline Female & $1.039(0.881-1.225)$ & 0.648 & & \\
\hline Pathology Grade & & 0.039 & & 0.067 \\
\hline Well & Reference & - & Reference & - \\
\hline Moderately differentiated & $1.253(0.957-1.642)$ & 0.012 & $1.051(0.832-1.142)$ & 0.32 \\
\hline Poorly & $1.248(0.941-1.656)$ & 0.03 & $1.121(0.901-1.456)$ & 0.193 \\
\hline Undifferentiated & $1.938(0.706-5.323)$ & 0.63 & $1.438(0.876-6.23)$ & 0.43 \\
\hline Year of diagnosis & & & & 0.000 \\
\hline 2004-2009 & Reference & - & Reference & - \\
\hline 2010-2015 & $2.062(1.744-2.438)$ & 0.000 & $2.056(1.737-2.433)$ & \\
\hline Tumor size & & & & $0 . .018$ \\
\hline$\leq 2 \mathrm{~cm}$ & Reference & - & Reference & - \\
\hline$<=3 \mathrm{~cm}$ & $1.224(1.004-1.492)$ & 0.045 & 1.187(0.969-1.455) & 0.098 \\
\hline$<=5 \mathrm{~cm}$ & $1.159(0.927-1.448)$ & 0.035 & $1.284(0.858-1.546)$ & 0.045 \\
\hline$>5 \mathrm{~cm}$ & $1.435(1.019-2.019)$ & 0.039 & $1.76(1.018-1.978)$ & 0.037 \\
\hline \multicolumn{5}{|l|}{ T stage } \\
\hline T1 & Reference & - & Reference & - \\
\hline
\end{tabular}




\begin{tabular}{|lllll|} 
T2 & $1.354(1.028-1.784)$ & 0.031 & $1.249(0.943-1.655)$ & 0.012 \\
\hline T3 & $1.321(1.007-1.733)$ & 0.045 & $1.425(1.101-1.746)$ & 0.013 \\
\hline T4 & $1.602(1.213-2.117)$ & 0.001 & $1.737(1.23-2.443)$ & 0.009 \\
\hline
\end{tabular}

Table 3

Univariate and Multivariate cox regression analysis of ampulla cancer patients for CSS 


\begin{tabular}{|c|c|c|c|c|}
\hline Variables & Univariate analysis & & \multicolumn{2}{|l|}{ Multivariate analysis } \\
\hline \multicolumn{2}{|l|}{ Age } & \multicolumn{3}{|l|}{0.000} \\
\hline$<50$ & Reference & - & Reference & - \\
\hline $50-70$ & $1.414(1.127-1.774)$ & 0.003 & $1.493(1.188-1.876)$ & 0.001 \\
\hline$>70$ & $1.752(1.388-2.211)$ & 0.000 & $1.896(1.498-2.4)$ & 0.000 \\
\hline Race & & 0.094 & & \\
\hline White & Reference & - & & \\
\hline Black & $1.252(0.998-1.57)$ & 0.052 & & \\
\hline Other & $0.938(0.787-1.117)$ & 0.470 & & \\
\hline \multicolumn{5}{|l|}{ Sex } \\
\hline Male & Reference & - & & \\
\hline Female & $0.906(0.799-1.027)$ & 0.122 & & \\
\hline Pathology Grade & & 0.000 & & \\
\hline Well & Reference & - & Reference & - \\
\hline Moderately differentiated & $1.594(1.248-2.035)$ & 0.000 & $1.427(1.116-1.826)$ & 0.005 \\
\hline Poorly & $2.491(1.946-3.19)$ & 0.000 & $1.774(1.379-2.282)$ & 0.000 \\
\hline Undifferentiated & $2.519(1.261-5.034)$ & 0.009 & $1.283(0.636-2.587)$ & 0.486 \\
\hline \multicolumn{5}{|l|}{ Metastasis } \\
\hline No & Reference & - & Reference & - \\
\hline Yes & $2.416(1.79-3.26)$ & 0.000 & $1.564(1.146-2.136)$ & 0.005 \\
\hline \multicolumn{5}{|l|}{ Tumor size } \\
\hline$\leq 2 \mathrm{~cm}$ & Reference & - & Reference & - \\
\hline$<=3 \mathrm{~cm}$ & $1.305(1.126-1.513)$ & 0.000 & $1.059(0.911-1.23)$ & 0.458 \\
\hline$<=5 \mathrm{~cm}$ & $1.3(1.1-1.537)$ & 0.002 & $0.947(0.795-1.129)$ & 0.545 \\
\hline$>5 \mathrm{~cm}$ & $1.234(0.962-1.582)$ & 0.098 & $0.949(0.736-1.225)$ & 0.689 \\
\hline T stage & & 0.000 & & \\
\hline T1 & Reference & - & Reference & - \\
\hline T2 & $1.331(1.022-1.734)$ & 0.034 & $1.175(0.9-1.534)$ & 0.236 \\
\hline T3 & $2.554(1.993-3.273)$ & 0.000 & $1.87(1.448-2.415)$ & 0.000 \\
\hline
\end{tabular}




\begin{tabular}{|lllll|}
\hline T4 & $3.476(2.713-4.453)$ & 0.000 & $2.17(1.67-2.82)$ & 0.000 \\
\hline 0 & & & & \\
\hline $1-4$ & Reference & - & Reference & - \\
$>=5$ & $2.408(2.075-2.796)$ & 0.000 & $1.583(1.042-2.561)$ & 0.043 \\
Examined LNs count & $3.782(3.203-4.465)$ & 0.000 & $2.21(1.704-3.212)$ & 0.003 \\
$<=12$ & & & & \\
$>12$ & Reference & - & Reference & - \\
\hline Lymph node Metastasis & $0.819(0.724-0.927)$ & 0.002 & $0.692(0.609-0.786)$ & 0.000 \\
\hline NO & & & & \\
\hline Yes & Reference & - & Reference & - \\
\hline
\end{tabular}

Table 4

Accuracy of the prediction score of the nomogram and TNM stage for estimating prognosis of AC patients. 


\begin{tabular}{|lll|}
\hline & \multicolumn{2}{l}{ Value $(95 \% \mathrm{Cl})$} \\
\cline { 2 - 3 } Variable & Internal validation & External validation \\
\hline C index for nomogram & $0.686(0.584-0.773)$ & $0.713(0.651-0.784)$ \\
\hline $\begin{array}{l}\text { C index } \\
\text { (bootstrap corrected) }\end{array}$ & 0.674 & 0.698 \\
\hline & & \\
\hline C index for TNM stage & $0.616(0.533-0.754)$ & $0.647(0.551-0.719)$ \\
\hline C index & 0.615 & 0.643 \\
\hline (bootstrap corrected) & & \\
\hline & & \\
\hline 1 year AUC for nomogram & $0.719(0.634-0.781)$ & $0.698(0.625-0.784)$ \\
\hline 3 year AUC for nomogram & $0.788(0.672-0.833)$ & $0.764(0.709-0.811)$ \\
\hline 5 year AUC for nomogram & $0.782(0.653-0.858)$ & $0.774(0.701-0.813)$ \\
\hline & & \\
\hline 1 year AUC for TNM stage & $0.596(0.537-0.702)$ & $0.658(0.605-0.704)$ \\
\hline 3 year AUC for TNM stage & $0.673(0.601-0.714)$ & $0.717(0.659-0.801)$ \\
\hline 5 year AUC for TNM stage & $0.689(0.633-0.768)$ & $0.677(0.601-0.713)$ \\
\hline
\end{tabular}

Figures 
A

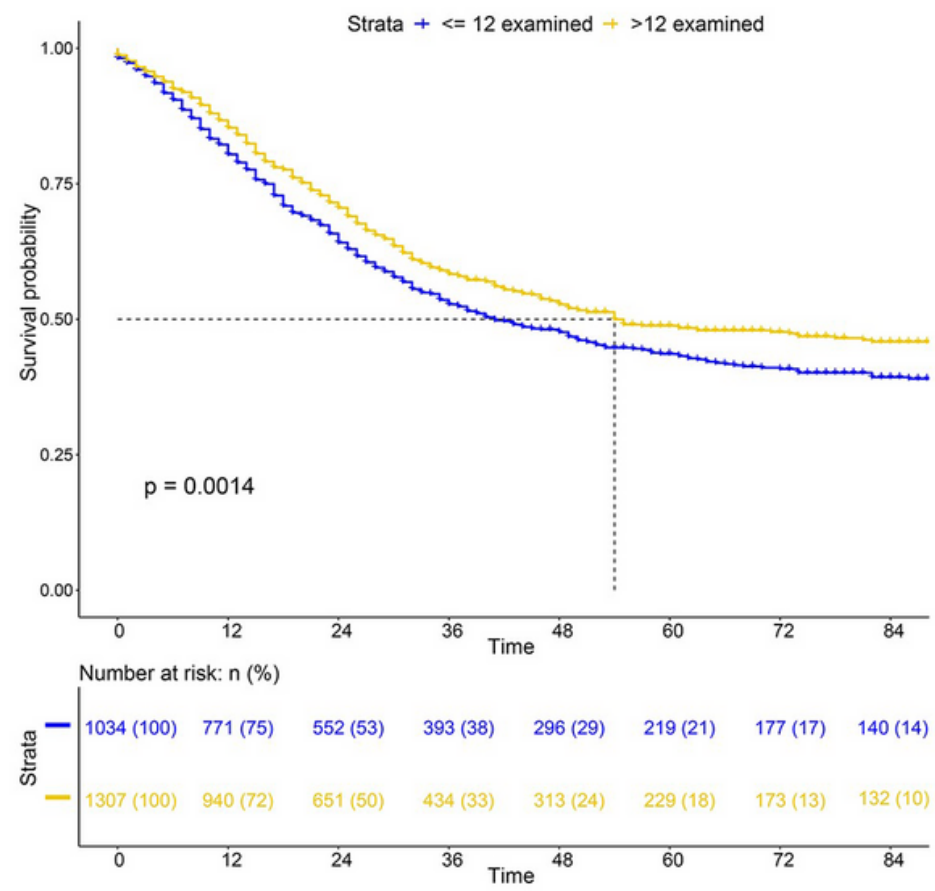

B

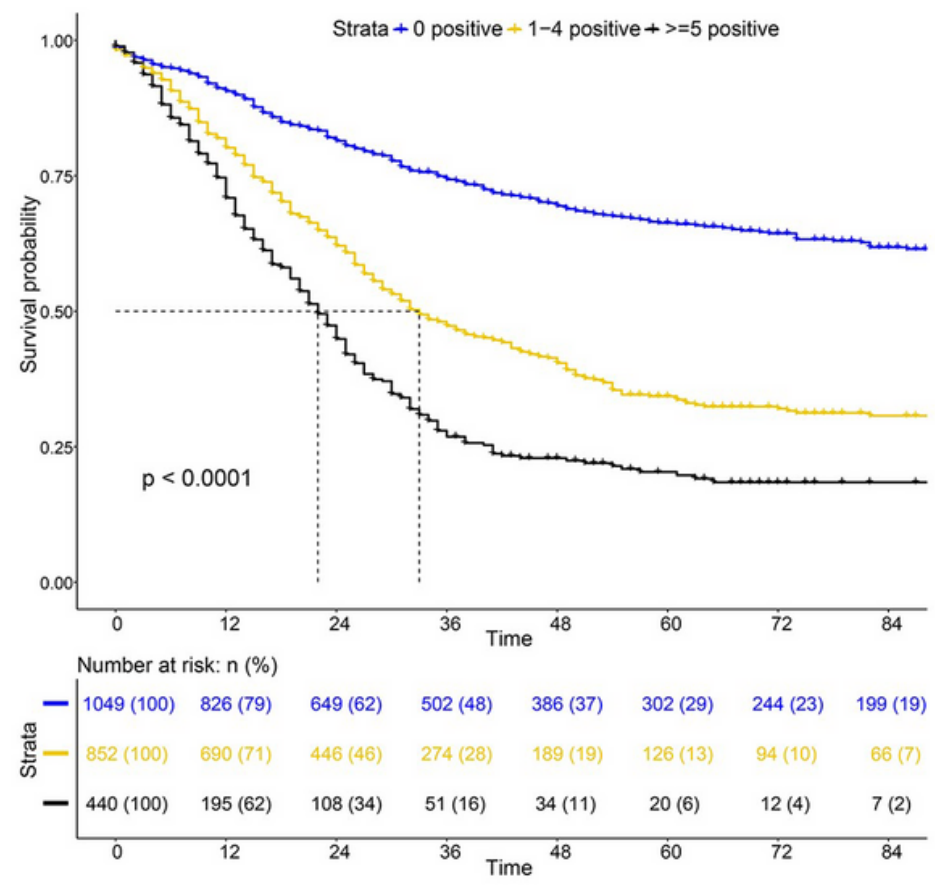

Figure 1

Kaplan-Meier survival analysis according to the number of examined LNs and positive LNs. (A) examined LNs, (B) positive LNs. 


34

$4 \quad 5$

$5 \quad 6$

$6 \quad 7$

$7 \quad 8$

8

Age

T stage

M stage

Grade

Examined nodes count

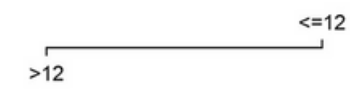

Total Points

1-year survival

3-year survival

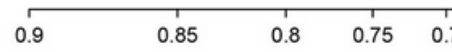

0.9

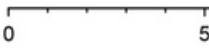

$\mathrm{T} 2$

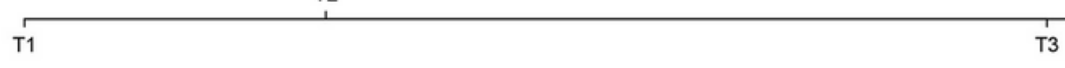

M0

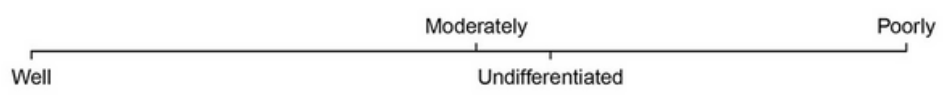

$<=12$

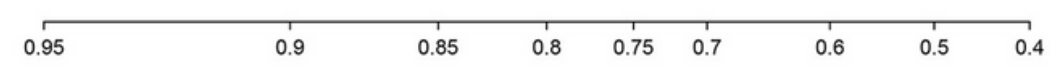

5-year survival

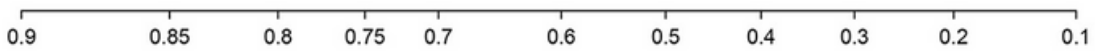

Figure 2

Nomogram predicted 1-, 3- and 5-year cancer specific survival for patients with resected AC. 
A

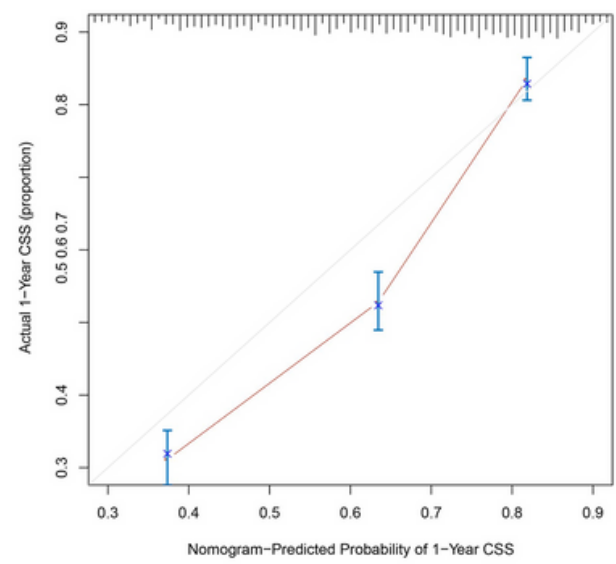

B

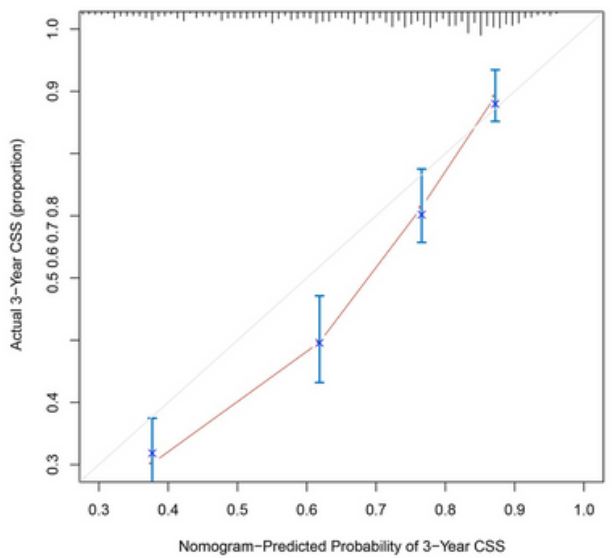

C

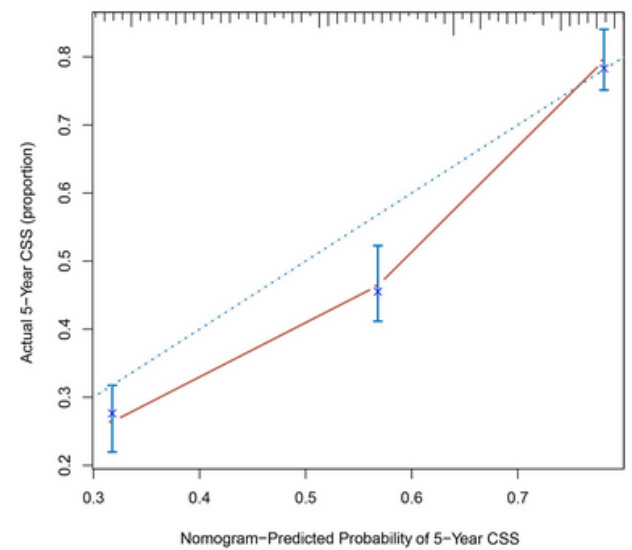

\section{Figure 3}

The calibration curves at 1-, 3- and 5-year point in the 2004-2015 cohort. (A) 1-year survival. (B) 3-year survival. (C) 5-year survival. Nomogram-predicted CSS is plotted on the x-axis; cancer specific survival is plotted on the y-axis.

A

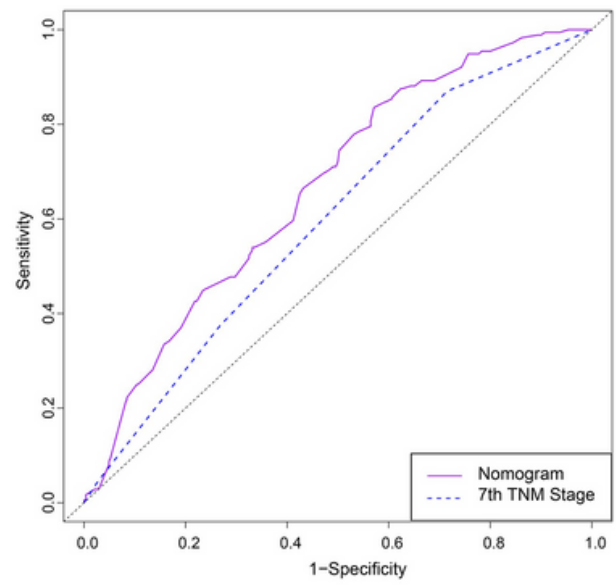

D

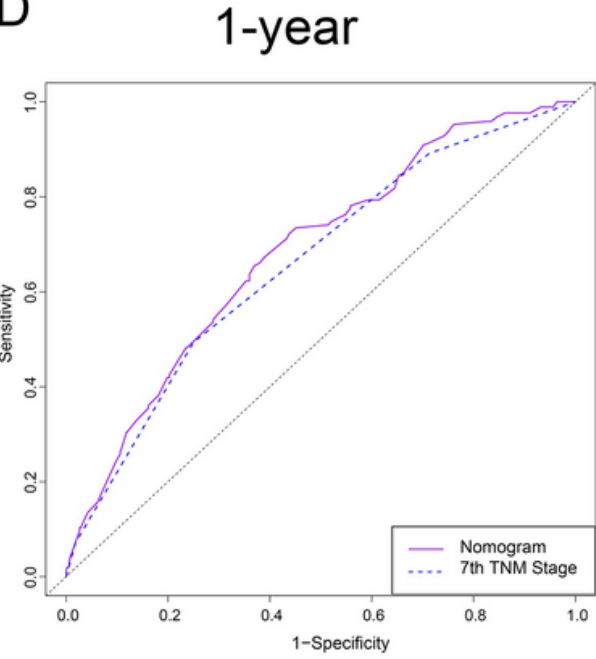

B

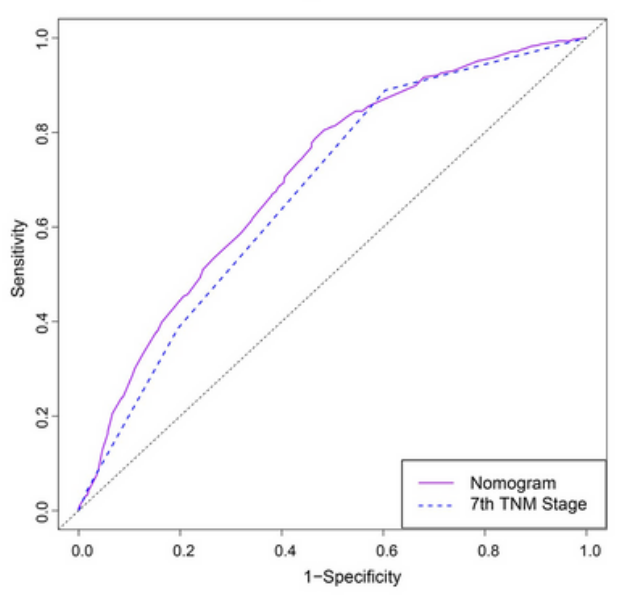

E

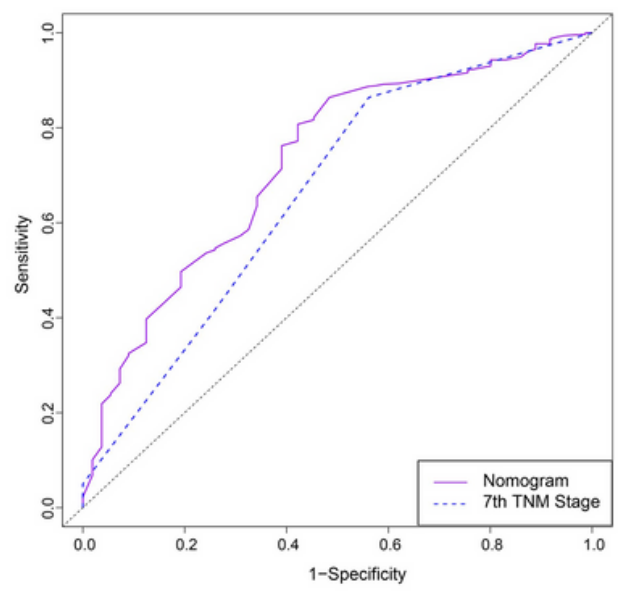

C 5-year

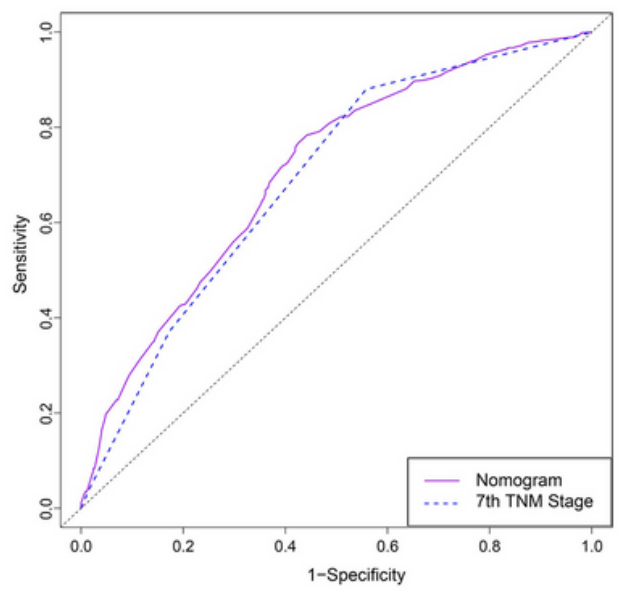

F

5-year

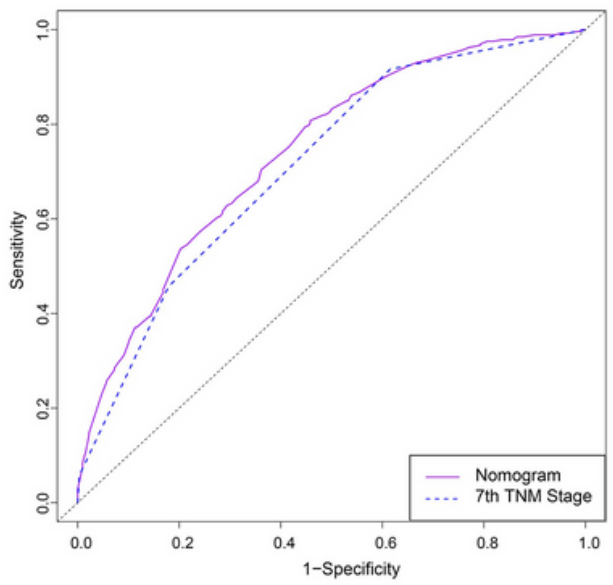


Figure 4

ROC curve of the Nomogram and 7th TNM Stage in prediction of prognosis of patients from 2004 to 2015. (A-C) ROC curve for 1-, 3- and 5-year point in 2004-2009 cohort. (D-F) ROC curve for 1-, 3- and 5year point in 2010-2015 cohort.

A
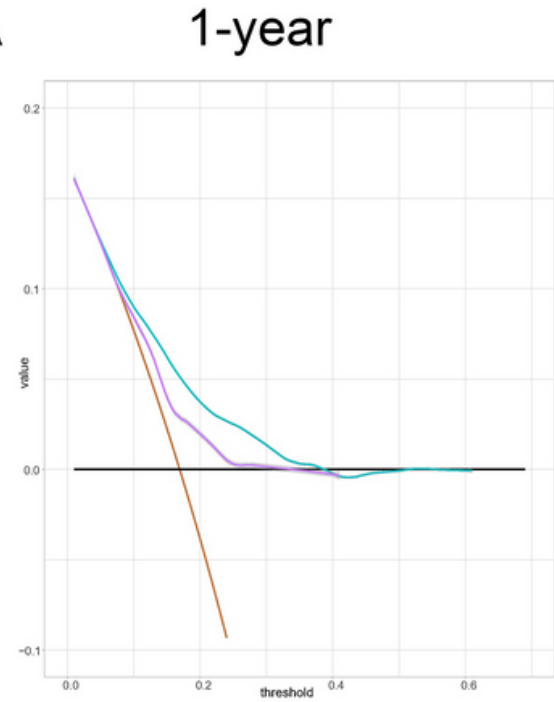

D
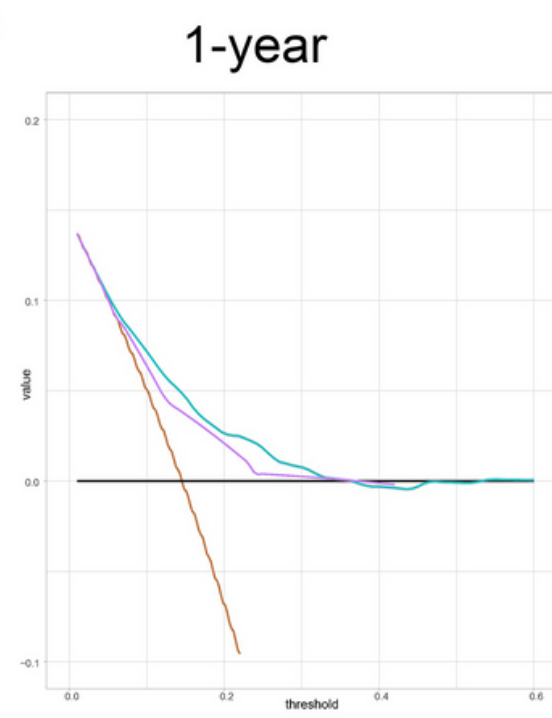

B

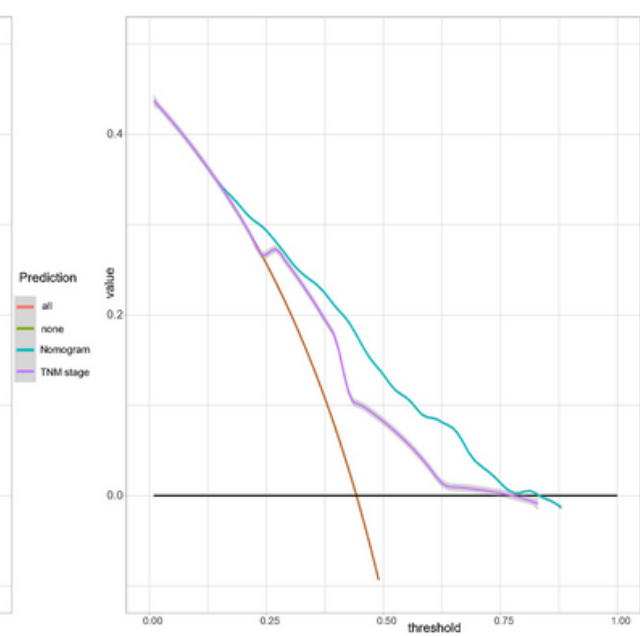

$\mathrm{E}$

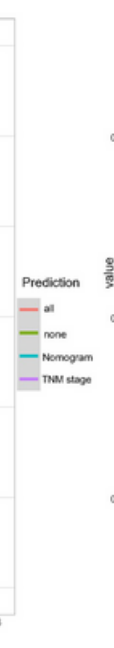

3-year
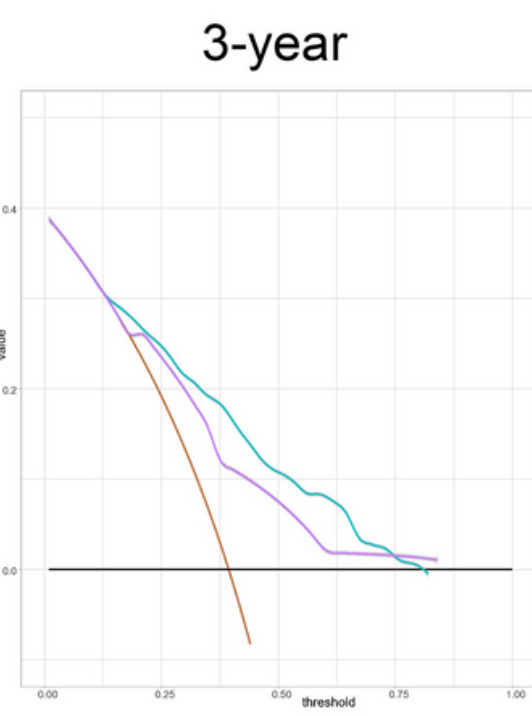

C 5-year
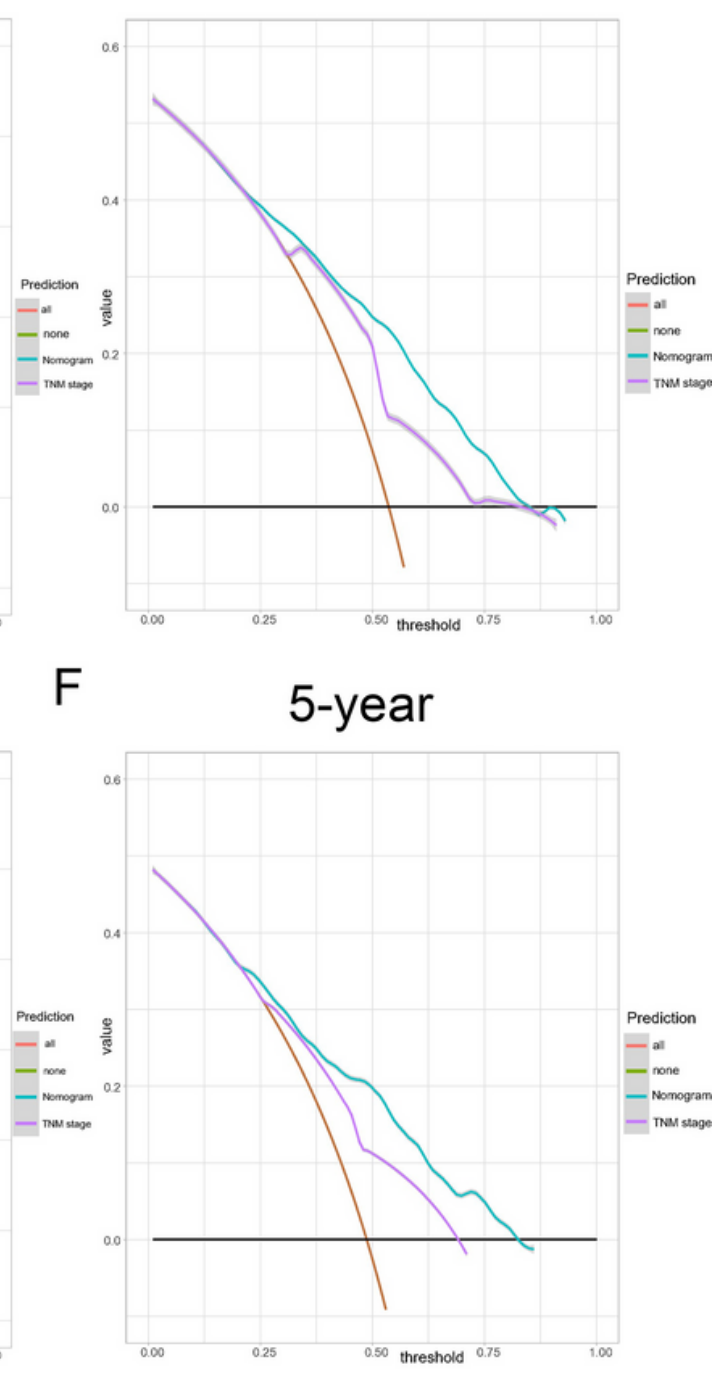

Figure 5

Decision curve analysis for the Nomogram and the model 7th TNM Stage in prediction of prognosis of patients. (A-C) 1-, 3- and 5- year point in 2004-2009 cohort. (D-F) 1-, 3- and 5-year point in 2010-2015 cohort.

\section{Supplementary Files}

This is a list of supplementary files associated with this preprint. Click to download.

- supplementary.SupplementaryFigure1.docx 